\title{
El aprendizaje cooperativo como metodología para la formación por competencias en la carrera de Derecho
}

\section{Cooperative learning as a methodology for competency- based training in law degree}

\author{
Yenny Pinto Sarmiento ${ }^{1}$
}

\begin{abstract}
Resumen: la formación por competencias ha sido vista como una buena fórmula para mejorar la calidad del aprendizaje en los estudiantes, dado que se potencia su nivel de participación en el proceso de enseñanzaaprendizaje. El artículo cuenta la experiencia llevada a cabo en el curso Derecho del Trabajo I, de la Universidad de Antofagasta, que se desarrolló bajo la metodología llamada de Aprendizaje basado en problemas (ABP), que fomenta el aprendizaje cooperativo a través de la simulación de una situación de demanda laboral, para lo cual se dividió al curso entre demandantes, demandados y tribunal; debiendo cada grupo asignar roles y funciones hasta llegar a la audiencia de juicio.
\end{abstract}

Palabras clave: formación por competencias, aprendizaje basado en problemas, enseñanza del Derecho.

Abstract: competency-based training has been seen as a good way to improve the quality of student learning to the extent that maximizes its level of participation in the process of teaching -learning. This paper shows the experience carried out in the subject of Labour Law I of Universidad de Antofagasta and was developed under the methodology called problem-based Learning (ABP in Spanish). ABP promotes cooperative learning by simulating a labour demand situation, where class group is divided between plaintiffs, defendants and court. Each group must assign roles and functions, even the hearing.

Key words: competency-based training, problem-based Learning, law teaching.

\section{Introducción: el diagnóstico}

La Facultad de Ciencias Jurídicas de la Universidad de Antofagasta fue visitada en el mes de abril del presente año por la agencia acreditadora, que requirió la elaboración de un informe de autoevaluación que reflejara de forma fidedigna nuestras fortalezas y debilidades. Dentro de las debilidades detectadas, se encontraba la falta de competencias básicas con las que llegaban los alumnos de primer año de la carrera, lo que traía aparejado el problema de las tasas de retención del primer año y demora en el tiempo de titulación. A esto debemos sumar que la Universidad de Antofagasta, en su modelo pedagógico, declara expresamente que el enfoque curricular adoptado por la Universidad promueve una formación centrada en el aprendizaje basado en competencias.

\footnotetext{
${ }^{1}$ Profesora de Derecho del Trabajo y Derecho Comercial, Universidad de Antofagasta; DEA en Derecho laboral, Universidad de Deusto, España; Máster en Derecho de la Empresa, Universidad de Deusto, España. Correo electrónico: ypinto@ucn.cl
} 
Como ya es conocido, varias universidades del país, y especialmente carreras de Derecho, se encuentran en los últimos años en un proceso de rediseño curricular, por lo que ha sido necesario formular nuevos planes de estudio basados $\mathrm{o}$ en formación por competencias, y así ajustarse a las tendencias mundiales en temas de educación. La educación por competencia ha sido vista como una fórmula para mejorar el aprendizaje del estudiante, acercando la brecha existente entre la educación universitaria y la práctica profesional. Ahora, el aprendizaje no se traduce sólo en la memorización de un texto, ni se reduce a la clase magistral del docente, sino que se centra desde la experiencia, permitiendo colaborar, expresarse y relacionarse, pero tomando al estudiante como centro y al profesor un guía en este camino ${ }^{2}$.

Este replanteamiento ha adoptado varias formas una de las principales está representada por el proyecto Tuning, del año 2003, que permite reflexionar sobre los diversos métodos de enseñanza-aprendizaje, a lo que debemos sumar la creación del Espacio Europeo de Educación Superior (EEES) ${ }^{3}$.

En este contexto, el docente adquiere un nuevo perfil, modificando nuestra actitud sobre cómo conducir la enseñanza, transformándose en un guía, orientando la búsqueda por parte del estudiante, promoviendo su desarrollo integral y desarrollando escenarios, procesos y experiencias de aprendizaje significativo y relevante, para de esa manera preparar de mejor forma al estudiante para el futuro.

Por ello, una de las interrogantes a resolver a la hora de implementar este nuevo plan es ¿cuáles serán los procedimientos metodológicos que mejor se ajustan a la carrera de Derecho?; y, ¿cuáles son las debilidades de nuestros alumnos?

Para el desarrollo del presente artículo, describiremos las técnicas de implementación del aprendizaje colaborativo en los cursos de Derecho del Trabajo I y Derecho Comercial en la Facultad de Derecho de la Universidad de Antofagasta.

\footnotetext{
${ }^{2}$ Como señalan IRIGOYEN, JIMÉNEZ Y ACUÑA : "La formación universitaria que parte de modelos basados en una concepción del conocimiento y de los contenidos como los objetivos primordiales del aprendizaje está siendo modificada, debido al acelerado cambio de los conocimientos, el acceso y la manera en como fluye $y$, en consecuencia, la provisionalidad de los saberes, generando que los procesos de adquisición, clasificación, disponibilidad, uso y generación del conocimiento demanden nuevos lenguajes y requieran un cambio de concepción con respecto a los saberes". (IRIGOYEN, Juan José; JIMÉNEZ, Miriam Yerith y ACUÑA, Fabiola. "Competencias y Educación Superior", En: Revista Mexicana de Investigación Educativa. 2011, vol. 16, núm. 48. P. 244.

${ }^{3}$ Véase: YÁNIZ, Concepción. "Las competencias en el currículo universitario: implicaciones para diseñar el aprendizaje y para la formación del profesorado". En: Red U. Revista de Docencia Universitaria. 2008, núm. 1(monográfico). Disponible en web: www.redu.m.es/Red_U/m1 (consultado el 1 de marzo de 2012).
} 


\section{Metodologías de enseñanza-aprendizaje}

Las nuevas metodologías de enseñanza aprendizaje se insertan en el contexto de la formación por competencia, como hemos visto. Aun cuando el concepto no resulta pacífico ${ }^{4}$, constituye el motor que alimenta el nuevo enfoque que viene comentándose.

Ello no quiere decir, sin embargo, que la clase magistral deba desaparecer, pues como indica Tobón, entre las razones para acoger la formación por competencias, se encuentran: permite una gestión de calidad mediante la evaluación de la calidad de desempeño y la evaluación de la formación; en segundo lugar, señala y con razón, que la formación por competencias se propone como política clave de educación superior en el ámbito internacional ${ }^{5}$.

\section{Entendemos por métodos docentes:}

un conjunto de decisiones sobre los procedimientos a emprender y sobre los recursos a utilizar en las diferentes fases de un plan de acción que, organizados y secuenciados coherentemente con los objetivos pretendidos en cada uno de los momentos del proceso, nos permiten dar una respuesta a la finalidad última de la tarea educativa.

Dentro de los métodos de enseñanza podemos encontrar una amplia gama que mezclan dos áreas como son: las actividades presenciales, como aquellas que reclaman una intervención directa de profesores y alumnos; y, en segundo lugar, las modalidades no presenciales, donde se engloban las actividades que los alumnos puedan realizar libremente ya sea en forma individual o por trabajo en grupo.

En cuanto a los métodos de enseñanza, existe una gran variedad, aunque entre los principales podemos mencionar los siguientes ${ }^{6}$ :

a) Método expositivo o lección magistral, cuya finalidad es transmitir conocimientos y activar procesos cognitivos en el estudiante.

\footnotetext{
${ }^{4}$ Hay varios conceptos de competencia y cierto es que éste depende, igualmente, de las distintas visiones del proceso enseñanza-aprendizaje, pero hay acuerdo en que supone la integración, no la reducción de la enseñanza a la transmisión de conocimientos, ni tampoco el mero desarrollo de habilidades. Sobre el concepto de competencia, señala YÁNIZ, Ibíd., que se define comúnmente como el conjunto de conocimientos, actitudes y habilidades necesarias para desempeñar una ocupación dada. Agrega: "Desde la perspectiva de la formación universitaria, destaca la idea de que para realizar con éxito un función determinada, se requieren conocimientos, actitudes y habilidades, lo cual cuestiona ideas subyacentes a algunas creencias difundidas en este nivel educativo, como por ejemplo, que con una formación únicamente ocupada de conocimientos se puede lograr una práctica adecuada en un campo complejo, que las actitudes son impropias de la formación universitaria o que las habilidades tienen que ver más con aptitudes personales que con el aprendizaje". Como señalan IRIGOYEN, JIMÉNEZ y ACUÑA (Ibíd. P. 247), el concepto más generalizado de competencia se refiere a un saber hacer en un contexto, definiendo el saber hacer no sólo en sentido de instrumentalidad, sino como un desempeño integrado de conocimientos (teórico, práctico o ambos), afectividad, compromiso, cooperación y cumplimiento.

${ }^{5}$ TOBÓN, Sergio. "El enfoque complejo de las competencias y el diseño curricular por ciclos propedéuticos". En: Acción Pedagógica. 2007, núm. 16. Pp. 14-28.

${ }^{6}$ Seguimos, en esta materia a MIGUEL DÍAZ, Mario de (Dir.); ALFARO ROCHER, Ignacio Javier; PODACA URQUIJO, Pedro; ARIAS BLANCO, José Miguel; GARCÍA JIMÉNEZ, Eduardo; LOBATO FRAILE, Clemente y PÉREZ BOULLOSA, Alfredo. Modalidades de enseñanza centradas en el desarrollo de competencias. Oviedo: Ministerio de Educación y Ciencia-Universidad de Oviedo, 2005. P. 83.
} 
b) Estudio de casos, cuya finalidad es la adquisición de aprendizajes mediante el análisis de casos reales o simulados.

c) Resolución de ejercicios y problemas, cuya finalidad es ejercitar, ensayar y poner en práctica los conocimientos previos.

d) Aprendizaje Basado en Problemas (ABP). Persigue desarrollar aprendizajes activos a través de la resolución de problemas.

e) Aprendizaje orientado a proyectos. Tiene por finalidad la realización de un proyecto para la resolución de un problema, aplicando habilidades y conocimientos adquiridos.

f) Aprendizaje cooperativo, cuya finalidad es desarrollar aprendizajes activos y significativos de forma cooperativa.

Sin embargo, no basta con la elección del método, ya que independiente de cuál sea la opción metodológica por la que se decida, se debe especificar en la planificación cuáles son las tareas específicas que se realizarán a lo largo del curso; aún más, es indispensable determinar claramente cuáles serán las tareas a realizar por el profesor y los alumnos antes, durante y después de la ejecución de cada uno de ellas, para que los alumnos tengan una referencia a la hora de planificar el trabajo que deberán realizar en forma autónoma.

\section{El aprendizaje cooperativo}

Una de las modalidades apropiadas para desarrollar otras competencias, desde nuestro parecer es el trabajo en grupo, donde una de las vertientes utilizadas es precisamente el aprendizaje cooperativo.

Entre las metodologías que hemos descrito, una de las que ha revestido mayor importancia encontramos el Aprendizaje Basado en Problemas (ABP) o Problem Based Learning (PBL), que cuenta con bastantes adeptos en diversas partes del mundo. Tiene sus raíces en la Universidad MacCaster, Canadá, en la Facultad de Ciencias de Ciencias de la Salud, en la década de los 60 y 70. Le siguieron las Escuelas de Medicina de Maastrich, en Holanda y Newcastle, Australia. En EEUU, lideraron esta formación, las universidades de New Mexico y Harvard, entre otras ${ }^{7}$. Como explican Roa y Romero, en la Universidad de MacCaster: “...existía un problema de salubridad pública determinado por las enfermedades de tipo respiratorio y que estaban aquejando a la población, sin una solución contundente del mismo. Por este motivo se tomó la adopción de problemas reales del entorno para desarrollar competencias pertinentes para dar solución a los problemas de salud",

\footnotetext{
${ }^{7}$ Véase MAC HEACHIE, Wilbert J. y SVINICKI, Marilla. Teaching Tips. Strategies, Research, and Theory for College and University Teachers. Boston, Nueva York: Houghton Mifflin Company, 2006. P. 221: "The ideas embodied in problem based-learning have a long history, ranging back at least to the use of cases in Harvard Medical School in the nineteenth century and extending through John Dewey's philosophy, Jerry Bruner's discovery learning, and the development in simulations in the 1960s".

${ }^{8}$ ROA BANQUEZ, Katherine y ROMERO GÓMEZ, Alexander. "Construcción de conocimiento con metodología de aprendizaje basado en problemas frente al modelo tradicional, en ambiente virtual". En: Revista Teoría y Praxis Investigativa. 2009. vol. 4, núm. 1. P. 12. El ABP, de acuerdo con Hernández y Lacuesta: “... Postman y Weingarner (1969) ya proponían un modelo de enseñanza que prescindiera de las
} 
El ABP puede definirse como el método en el que se utilizan problemas como punto de partida para la adquisición e integración de los nuevos conocimientos. Supone estructurar todo el módulo sobre la base del problema. Pedraz señala que: "es un modelo en el que el alumno construye su propio conocimiento, integrando objetivos de todas las disciplinas, con la ayuda de diferentes personas implicadas en el proceso de aprendizaje y de las herramientas bibliográficas adecuadas que las nuevas tecnologías le acercan"9.

Ahora bien, entre el ABP y el aprendizaje colaborativo no existen diferencias sustanciales, puesto que ambas admiten gradualidades y ello es porque el profesor, en la implementación de su estrategia pedagógica, debe ser consciente de sus propios medios, de sus posibilidades y la de sus estudiantes. Siguiendo a Duch, la misma metodología admite varios modelos, dependiendo precisamente de las disponibilidades de los académicos. Es decir, dependerá el número de alumnos, del nivel, del aula, de las materias ${ }^{10}$.

En este tipo de metodología el éxito de cada alumno depende de que el conjunto de sus compañeros alcancen las metas fijadas, por lo que la consecución de las metas requiere el desarrollo y despliegue de competencias relacionales que son clave en el desempeño profesional.

En lo que dice relación con los componentes más importantes del aprendizaje cooperativo, podemos mencionar los siguientes:

1. Interdependencia positiva: cada miembro percibe con claridad el vínculo con los compañeros de tal manera que uno no puede tener éxito si todos los demás no lo tienen.

2. Responsabilidad individual: cada alumno no sólo responde de su propio aprendizaje sino también del de sus compañeros.

3. Interacción cara a cara: la dinámica de la tarea implica interacciones continuas y directas entre los miembros.

4. Habilidades inherentes a pequeños grupos: el alumno debe adquirir, desarrollar y emplear habilidades básicas de trabajo en grupo.

5. Evaluación de los resultados y del proceso: el grupo debe desarrollar actividades de reflexión y evaluación del trabajo en grupo.

En este tipo de metodología el profesor adopta un rol de facilitador, lo que permite reforzar la confianza de los alumnos en su capacidad de trabajo autónomo y resolución de problemas. En el caso de los alumnos dentro de cada equipo se deben determinar roles, donde existen roles fundamentales como son el coordinador, secretario y facilitador.

clases magistrales y desarrollase la capacidad creativa de los estudiantes mediante el planteamiento de preguntas y problemas abiertos...". (HERNÁN DEZ TRASOBARES, Alejandro y LACUESTA GILABERTE, Raquel. "Aplicación del aprendizaje basado en problemas (PBL) bajo un enfoque multidisciplinar: una experiencia práctica”. En: AYALA CALVO, Juan Carlos (coord.). Conocimiento, innovación y emprendedores: Camino al futuro. La Rioja: Ediciones Universidad de La Rioja, 2007. P. 32.)

${ }^{9}$ PEDRAZ, A. "Un método de aprendizaje para toda la vida: el ABP en el Espacio Europeo de la Educación Superior". En: PEDRAZ, A. (coord.). El aprendizaje basado en problemas: una herramienta para toda la vida. Madrid: Agencia Laín Entralgo, 2004. P. 11.

${ }^{10}$ DUCH, Barbara. "Modelos para la instrucción basada en problemas en cursos de pregrado". En: GROH, Susan, ALLEN, Deborah E. y DUCH, Barbara (eds). El poder del aprendizaje basado en problemas. Una gruía práctica para la enseñanza universitaria. Trad. de César Mendoza Loyola. Lima: Ediciones Pontificia Universidad Católica del Perú, 2006. Pp. 56-61. 


\section{Descripción del aprendizaje cooperativo en el curso de Derecho del Trabajo y Derecho Comercial}

Como ya se ha señalado, nuestros alumnos que ingresan a la Universidad de Antofagasta carecen de competencias básicas, como son la expresión oral o escrita; no saben trabajar en equipo, no tienen técnicas adecuadas de estudio, falta de espíritu crítico, etc. La metodología que describiremos, permite alcanzar el desarrollo de tales competencias

Para la implementación de las metodologías, los alumnos deben dividirse en equipos de 6 a 8 integrantes y designar roles dentro de cada equipo, además el profesor hará las veces tutor o facilitador, promoviendo la discusión en las sesiones de trabajo. Éste no será la autoridad del curso, ya que los alumnos solo se apoyaran en él para la búsqueda de información. Más bien, en este tipo de metodología, el trabajo de los alumnos es mayoritariamente autónomo, donde el profesor debe reforzar la confianza de los alumnos en su capacidad de aprendizaje y resolución de problemas, tal como se ha analizado en los presupuestos teóricos de la metodología.

En el caso del curso de Derecho del Trabajo I, separamos los alumnos en grupos de demandantes, demandados y tribunal, ya que el objetivo de la actividad es precisamente la simulación de un procedimiento laboral. Una vez determinados los equipos, los alumnos deberán designar los roles dentro de éste. En nuestro curso se ha determinado que en cada equipo debe haber un presidente, secretario, y un coordinador, que será quien se relacionará fuera de las sesiones con el profesor, y quien deberá, además, realizar las consultas y entregar los informes de avances que deben ir elaborando luego de cada sesión de trabajo.

En el caso de la presente implementación, se tomó la decisión de que el primer paso sería entregar toda la información necesaria a los alumnos y así evitar la incertidumbre y desconfianza a los alumnos que no estaban acostumbrados a realizar este tipo de técnicas, sino más bien a clases tradicionales centradas en el profesor. Es por esto que se les entregó un documento con la información de la metodología utilizada, con sus fundamentos, características y las competencias que desarrolla su implementación.

Además se entregó y analizó un documento donde se señala los pasos a seguir, como la designación de roles dentro de cada equipo, la planificación de la actividad, que incorporaba los resultados esperados en cada sesión, los requisitos con que deben cumplir la demanda, contestación y sentencia. También se les explicó la primera tarea a realizar en la primera clase, que consistía en dictar sus propias normas de convivencia.

Un tercer documento contiene el resultado de aprendizaje, que en este caso es "identificar las diversas etapas del procedimiento laboral". Entendemos por resultados de aprendizaje, a efectos de aplicación de esta metodología, Los resultados de aprendizaje son enunciados acerca de lo que se espera que un estudiante deba saber, comprender y / o ser capaz de demostrar una vez finalizado el proceso de aprendizaje. Los alumnos recibieron también las rúbricas que se utilizarían para la evaluación del trabajo y así de esa manera los alumnos conocieran de antemano los parámetros que se tomarían en cuenta en su evaluación. 
La primera tarea del profesor para la implementación de la metodología es la de elaborar o seleccionar una situación problema atractivo para los alumnos que permita desarrollar las competencias previstas en el programa que en nuestro caso serían las competencias previstas para Derecho del trabajo, que en el caso del programa de Derecho del trabajo I de la Facultad de Ciencias Jurídicas de la Universidad de Antofagasta. No sólo se limitan a conocer y comprender conceptos dogmáticos, sino además se exige a los alumnos razonar, argumentar y resolver situaciones jurídicas con un adecuado manejo de las normas y principios del Derecho del trabajo, por lo que nos encontrábamos con objetivos que no eran logrados solo con una clase magistral.

En nuestro caso, consideramos que la mejor estrategia se traducía en no presentar el problema por parte del profesor. Para tal efecto, se eligieron a dos alumnos de niveles más altos de la carrera para que representaran al trabajador y empleador y que cada grupo correspondiente se entrevistara con quien sería su representado. Así, los alumnos además de tomar conocimiento del problema, debían solicitar los documentos y pruebas que necesitarían para la posterior defensa dentro del juicio.

Abordado con esta perspectiva, se manifiesta una de las ventajas de este método, es decir, que permite desarrollar todas las fases de la enseñanza, comenzando por la exploración y culminando con la evaluación del mismo. Una vez conocido el problema, en la etapa de la asesoría, los alumnos pasan de la fase de la exploración a la fase de determinación y definición de los hechos de relevancia y los aspectos normativos aplicables. Se les exige examinar como grupo cuáles serían los componentes principales de la problemática, si el problema principal o primario genera otros problemas secundarios y cuáles serían las preguntas que deben resolver. Una vez determinado lo anterior, se pasa a otra fase del aprendizaje que es la planificación de la investigación, donde los alumnos por equipo deciden qué material necesitan, qué es lo que deben buscar y la forma de repartir las tareas.

En nuestra experiencia, en esta etapa los alumnos por primera vez asumían la responsabilidad de buscar ellos mismos la información que necesitaban, ya que hasta ese momento tenían la costumbre de que era el profesor quien les proporcionaba toda la información para la decisión de un supuesto problemático, memorizando los contenidos otorgados por el profesor.

Luego de la búsqueda de información, corresponde analizar todo lo que se ha recopilado como equipo, en cuanto a su pertinencia en relación al problema y si es necesario buscar más información que la obtenida hasta ese momento. Para analizar la pertinencia o no de lo recopilado hasta ese momento, se les pidió efectuar un check-list con el apoyo del profesor, en el que participa todo el curso, pudiendo así los alumnos corroborar si la información obtenida es pertinente y si necesitan de una mayor búsqueda, para continuar con las actividades siguientes.

Posteriormente, se les exigió efectuar una síntesis, es decir, se les pidió determinar el diagnóstico, las posibles soluciones y argumentos a utilizar. Para concluir esta etapa, se efectuó una lluvia de ideas, en la que participamos como facilitadores para el análisis crítico de las soluciones.

Una vez determinadas las soluciones y argumentos se deben elaborar los escritos correspondientes: en primer lugar, una demanda y dentro de un plazo, una contestación. En 
esta etapa solicitamos a los equipos exponer en power point los escritos correspondientes, participando todos los alumnos en efectuar correcciones a los errores cometidos, logrando que de esa manera todos lograran una importante retroalimentación.

Posteriormente, solicitamos al grupo que representaba al tribunal la fecha de la audiencia preparatoria, dictando la resolución correspondiente, la que debía además ser notificada en la forma que correspondía. En esta fase del trabajo sería ideal realizar en primer lugar la audiencia preparatoria y luego de un tiempo la de juicio, para así simular de mejor manera el procedimiento real; sin embargo, nos encontramos con la dificultad de que no es muy extenso el tiempo con que se cuenta para realizar estas actividades, para no descuidar los contenidos del curso.

Es por lo anterior que se debieron realizar ambas audiencias en el mismo día. En primer lugar, se realizó la audiencia preparatoria y luego la audiencia de juicio. En dicha audiencia, los alumnos rindieron la prueba: presentaron documentos y además llevaron personas externas como testigos y videos en defensa de cada representado. Por su parte, el equipo que representaba al tribunal preparó un blog que contenía todas las informaciones respecto a la constitución de éste y notificaciones correspondientes, además de realizar un panel en dependencias de la Facultad que hizo las veces de estado diario. Un integrante de dicho equipo asumió el rol de actuario, tomando nota de lo obrado y efectuando el llamado a las partes.

Durante el desarrollo de la audiencia, uno de los aspectos a destacar fue la motivación y preparación por parte de los alumnos, visualizando el desarrollo de las competencias relacionadas directamente con los objetivos del curso y a su vez con el perfil de egreso declarado por nuestra carrera, como son la argumentación, la expresión oral y escrita, la redacción jurídica, la defensa de intereses ajenos, y la correcta litigación en procedimientos orales, como exige la actual reforma laboral.

Posteriormente el tribunal dictó su sentencia, la que además dentro de un plazo debió ser presentada por escrito. Y, finalmente, se culminó con la fase de final de retroalimentación, sometiendo a discusión del curso cómo se llegó a la solución del problema, qué estrategias se utilizaron y qué aspectos teóricos, procedimentales y actitudinales convienen destacar, mejorar o corregir.

Para el caso del curso de Derecho comercial, una vez entregado el caso se les encargó a los alumnos redactar un informe jurídico con respuestas a las interrogantes dadas por el cliente, además de constituir no sólo la sociedad, sino crear una empresa, con la iniciación de actividades, permisos municipales, contratos de trabajo, marketing, etc. Las restantes técnicas fueron básicamente idénticas a las ya descritas.

En lo que dice relación con la evaluación, como se dijo anteriormente, los parámetros a evaluar por parte del profesor se dieron a conocer en un primer momento al entregar a los equipos las rúbricas utilizadas para la presente actividad. Pero además en el caso del aprendizaje colaborativo, los alumnos debieron realizar una autoevaluación y una coevaluación, permitiendo que los alumnos valoraran las fortalezas y debilidades individuales y de equipo. Para la coevaluación y autoevaluación los parámetros empleados fueron los siguientes: 
- Preparación: si el material utilizado resultaba de relevancia para la resolución del caso, si se aplicaron conocimientos previos, y si se demostró preparación para las sesiones de trabajo en grupo.

- Participación y contribuciones al trabajo en grupo: si tenía la capacidad de dar y aceptar retroalimentación constructiva.

- Habilidades interpersonales: si muestra habilidad para comunicarse con sus compañeros, es respetuoso, colaborativo y responsable.

- Evaluación crítica: define y analiza el problema, siendo capaz de generar una hipótesis, identificando los objetivos del aprendizaje.

\section{Conclusiones}

Consideramos que la metodología descrita presenta innegables ventajas, pero también algunas dificultades para los docentes. En cuanto a las ventajas, podemos señalar las siguientes:

- Permite analizar y resolver cuestiones propias de la práctica profesional, acercando a los estudiantes al tipo de problemas que tendrá que afrontar en el futuro.

- Facilita el aprendizaje de competencias complejas asociadas a la resolución de problemas, el trabajo en equipo o la toma de decisiones.

- Sitúa al estudiante ante situaciones cercanas al desarrollo de la profesión, que exigen de su capacidad de innovar, integrar y aplicar conocimientos y habilidades asociados a la titulación o incluso a otros campos del saber; y, por supuesto, le exige que aprenda a debatir y argumentar ante personas que tiene una formación similar a la suya.

- Fomenta el trabajo grupal e interprofesional.

Además debemos añadir que el trabajo en grupo cooperativo posee claras ventajas las que lógicamente influirán en el aprendizaje del alumno, ya que logra en la mayoría de los alumnos competencias que solo con la clase magistral no eran desarrolladas, como la comunicación, la interacción social, el aceptación de la diferencia, sin mencionar un factor muy relevante a nuestro parecer, que es la motivación con que los alumnos afrontan este desafío.

En cuanto a las dificultades, pueden señalarse, entre otras, que algunos alumnos aún no tenían ciertos conocimientos que se supone que ya habían adquirido con anterioridad en otras asignaturas, la desmotivación en un principio generada por la desconfianza por parte del alumnado, al no haber experimentado antes estas metodologías; otro problema fue la falta de infraestructura adecuada para trabajar en equipo, y por último, la falta de una adecuada base de datos para que los estudiantes pudieran obtener jurisprudencia.

Con todo, el balance es positivo, pues como hemos dicho permite al alumno adquirir varias competencias que no eran logradas con una clase magistral, pero a pesar de esto no se quiere decir que se debiese cambiar todo el sistema tradicional por estas nuevas metodologías, pues de acuerdo con nuestra experiencia es necesario complementarlas y así 
el alumno logre dichas habilidades pero con un conocimiento sólido de los contenidos de cada asignatura.

\section{Referencias bibliográficas}

MIGUEL DÍAZ, Mario de (dir.); ALFARO ROCHER, Ignacio Javier; APODACA URQUIJO, Pedro; ARIAS BLANCO, José Miguel; GARCÍA JIMÉNEZ, Eduardo; LOBATO FRAILE Clemente y PÉREZ BOULLOSA, Alfredo. Modalidades de enseñanza centradas en el desarrollo de competencias. Oviedo: Ministerio de Educación y Ciencia-Universidad de Oviedo, 2005. P. 83.

DUCH, Bárbara. "Modelos para la instrucción basada en problemas en cursos de pregrado". En: GROH, Susan, ALLEN, Deborah E. y DUCH, Bárbara. El poder del aprendizaje basado en problemas. Una gruía práctica para la enseñanza universitaria. Trad. de César Mendoza Loyola. Lima: Ediciones Pontificia Universidad Católica del Perú, 2006. Pp. 56-61.

HERNÁN DEZ TRASOBARES, Alejandro y LACUESTA GI LABERTE, Raquel. “Aplicación del aprendizaje basado en problemas (PBL) bajo un enfoque multidisciplinar: una experiencia práctica" En: AYALA CALVO, Juan Carlos (coord.). Conocimiento, innovación y emprendedores: Camino al futuro. La Rioja: Ediciones Universidad de La Rioja, 2007. P. 32.

IRIGOYEN, Juan José; JIMÉNEZ, Miriam Yerith y ACUÑA, Fabiola. "Competencias y Educación Superior". En: Revista Mexicana de Investigación Educativa. 2011, vol. 16, núm. 48. P. 244.

MACHEACHIE, Wilbert J. y SVINICKI, Marilla. Teaching Tips. Strategies, Research, and Theory for College and University Teachers. Boston, Nueva York: Houghton Mifflin Company, 2006. P. 221.

PEDRAZ, A., "Un método de aprendizaje para toda la vida: el ABP en el Espacio Europeo de la Educación Superior". En: PEDRAZ, A. (coord.). El aprendizaje basado en problemas: una herramienta para toda la vida. Madrid: Agencia Laín Entralgo, 2004. P. 11.

ROA BANQUEZ, Katherine, ROMERO GÓMEZ, Alexander. "Construcción de conocimiento con metodología de aprendizaje basado en problemas frente al modelo tradicional, en ambiente virtual”, En: Revista Teoría y Praxis Investigativa. 2009, vol. 4, núm. 1. P. 12.

TOBÓN, Sergio. "El enfoque complejo de las competencias y el diseño curricular por ciclos propedéuticos". En: Acción Pedagógica. 2007, núm. 16. Pp. 14-28.

YÁNIZ, Concepción. "Las competencias en el currículo universitario: implicaciones para diseñar el aprendizaje y para la formación del profesorado". En: Red U. Revista de Docencia Universitaria. 2008, núm. 1 (monográfico). Disponible en web: www.redu.m.es/Red_U/m1 (consultado el 1 de marzo de 2012). 